\title{
Inorganic materials for photovoltaics: Status and futures challenges
}

\author{
A. $\operatorname{Sladui}(*)$ \\ Laboratoire ICUBE, CNRS 83 University of Strasbourg \\ 23 rue du loess, 67037 Strasbourg, France
}

\begin{abstract}
Summary. - This paper review the present technologies for the fabrication of solar cells and modules based on the most common semiconductors namely silicon, CuInGaSe(S) and CdTe materials as well as on III-V concentrated photovoltaic cells and modules. For silion technology, we give insights on the growth of monocrystalline and multicrystalline silicon wafers and then we describe the most common solar cells designs and how to fabricate them. We also provide information about the fabrication of silicon modules and their performances. As for the thin-films solar cells, we present the structurale and optical properties of the CIGS and CdTe materials as well as the solar cell structures. The multi-junction concept cell that involves several III-V materials of different bandgaps is also described, and data on their fabrication, performances and mounting as modules are presented. Finally, a short outlook on the coming materials for solar cells is provided.
\end{abstract}

\section{1. - Introduction}

The technologies of photovoltaic cells and modules are numerous and call for diverse and varied materials and manufacturing processes $[1,2]$. They are traditionally classi-

$\left(^{*}\right)$ E-mail: Abdelilah.slaoui@unistra.fr 
fied into three categories related to their maturity: first-, second- and third-generation modules, the distinctions between these generations depends on the author! (fig. 1).

The so-called first-generation technologies include cells and modules made from wafers. This is the case for crystalline silicon and tandems made from III-V elements. Crystalline silicon ( $\mathrm{sc}-\mathrm{Si}$ ) technologies have dominated the market since the early 1960s [3,4]. Automation, upscaling, better light management, and Chinese manufacturing capabilities have lowered the cost of these modules to a level that it is making it difficult nowadays for competing technologies to gain market shares. This first generation of solar cells is now mature and the main improvement paths consist, on the one hand, of increasing the efficiency by using $n$-type silicon and by carrying out heterostructures with amorphous silicon or even perovskites and on the other hand, to reduce manufacturing costs by constructing "Giga-Factories" and reducing the silicon cell thickness. Besides, multi-junction cells based on III-V compounds have traditionally been developed for space applications since the 1990s and have been used for several years in concentration systems.

The second generation consists of thin-film modules of simple semiconductor materials (amorphous and micro or nano-crystalline silicon) [5] or compounds (CIGS and CdTe), whose manufacturing processes involve depositing a thin layer of active and absorbing semiconductor on an inexpensive substrate (glass, metal or polymer). Modules with amorphous silicon (a-Si) have been manufactured since the 1980s, whereas the CdTe ad CIGS modules appeared at the mid-2000s. For long time, the industry considered the thin films as a real technological breakthrough capable of taking a significant market share. However, the growth of the CdTe and CIGS sectors is delayed by the very low price of the crystalline silicon modules. The amorphous silicon based modules are currently experiencing problems of survival because its efficiencies are low and its cost remains high. It will not be described in detail here.

The third generation of solar cells brings together technologies that are under development that can mature in the medium to long term by lowering costs and/or increasing efficiencies [6]. It includes i) advanced architectures to achieve efficiencies that can theoretically exceed $60 \%$, ii) organic or hybrid thin films and iii) perovskites. For instance, thin hybrid films (a combination of inorganic and organic) were explored since the 90s, and the most studied structure was the so called "Grätzel" dye solar cells (or DSSC) that combine nanocrystalline anastase $\mathrm{TiO}_{2}$ with organic dyes [7-10]. Conversion efficiencies, initially very low, have taken off these last six years due to the use of perovskites as absorber materials. However, these compounds have a limited lifetime (several thousand hours) linked to the degradability of organic compounds by atmospheric agents (UV, O2, water vapor) and to the instability of the interfaces between the organic active layer and inorganic electrodes such as conductive transparent oxides or metals. One of the challenges will not only be to stabilize performance but also to find encapsulation methods to achieve appropriate lifetimes for the production of mass electricity. We will not report on those structures below.

In the following, we will describe the technologies for the fabrication of silicon modules, CIGS and CdTe modules as well as III-V concentrated photovoltaic cells. 


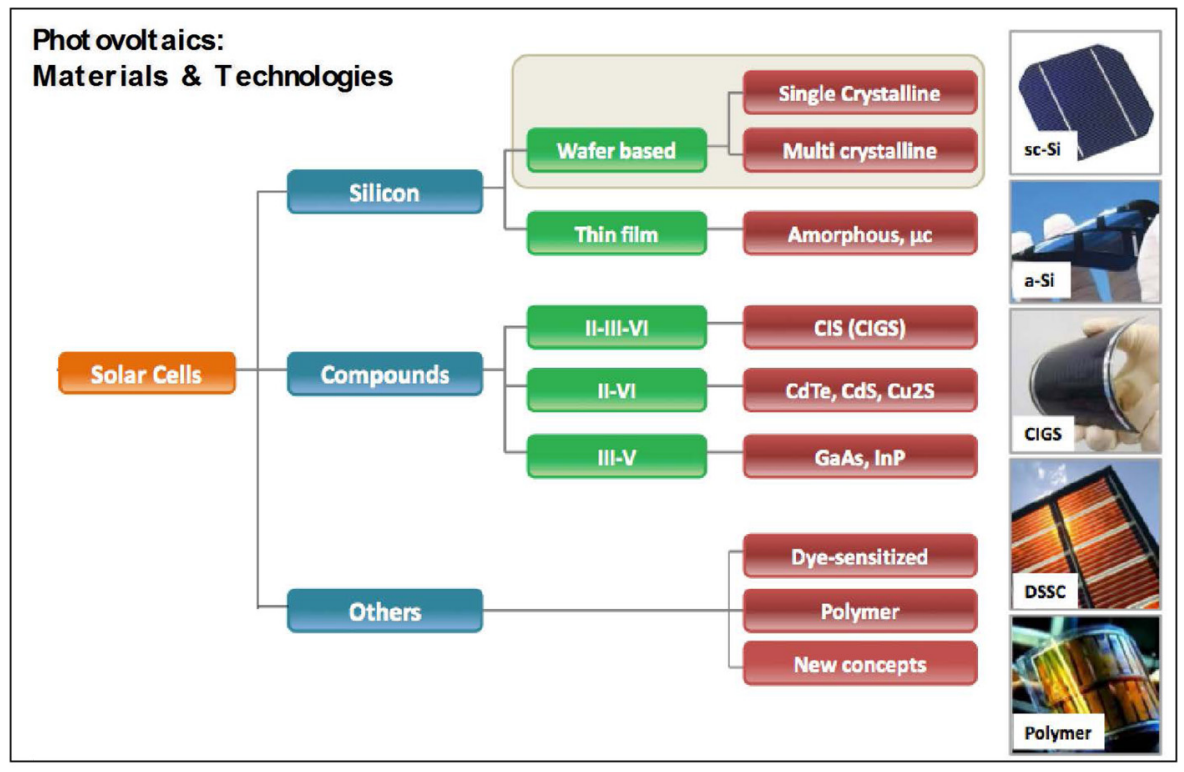

Fig. 1. - Materials and technologies used for photovoltaics.

\section{2. - Crystalline silicon modules}

The silica $\left(\mathrm{SiO}_{2}\right)$ found in the form of quartz is the basic material for producing silicon wafers for electronic and photovoltaic devices. The silica is reduced by carbon in an arc furnace to produce metallurgical silicon which, in turn, will have to be purified to obtain very high-purity silicon, called polysilicon. The latter is melted and solidified to produce ingots which can have different crystalline qualities according to the process used: monocrystalline silicon is obtained by drawing Czochralski or multicrystalline silicon by directional solidification. The ingots are then cut into bricks which themselves are sawn into thin wafers. The wafers make it possible to manufacture cells which are interconnected in series in sealed and mechanically resistant modules. The value chain for monocrystalline and multicrystalline silicon modules is shown in fig. 2 .

2'1. Single crystal silicon lingots. - The monocrystalline silicon ingots are produced in most cases by the Czochralski $(\mathrm{Cz})$ method. The polysilicon rocks are melted in a quartz crucible where they are placed in the presence of boron for the $\mathrm{p}$ doping of silicon with a typical concentration of $5 \times 10^{15}$ to $10^{16} \mathrm{~cm}^{-3}$ to reach resistivities of the order of 0.1 to $1 \Omega \cdot \mathrm{cm}$. A monocrystalline seed is then quenched in the bath of liquid silicon and removed very slowly by a simultaneous movement of rotation and translation, resulting in the growth of an ingot from the bath. For a material of very good crystalline quality, it is necessary to control the growth temperature $\left(\approx 1410^{\circ} \mathrm{C}\right)$, its uniformity and the speed of growth which should be slow and constant. The ingots are cylindrical due to the rota- 


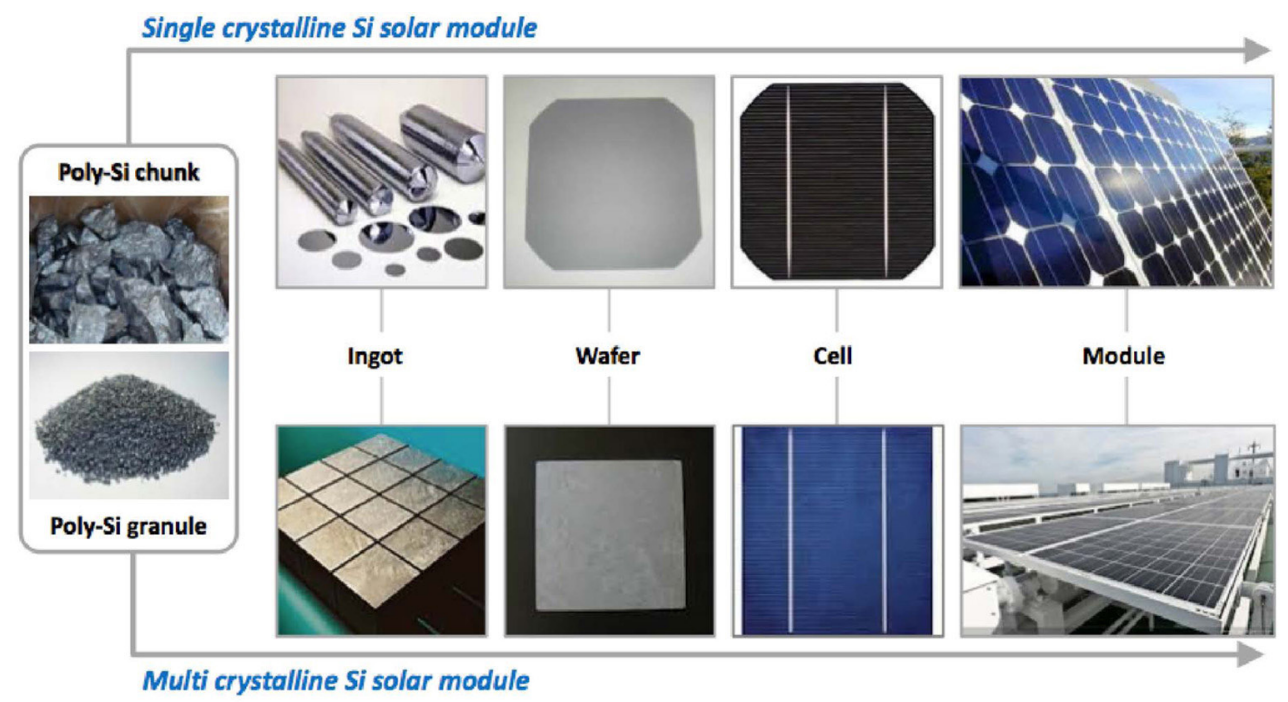

Fig. 2. - The value chain for the fabrication of monocrystalline (top) and multicrystalline (bottom) silicon photovoltaic modules.

tional movement. They have a diameter of between 10 and $30 \mathrm{~cm}$ nowadays, a length of 1 to 2 meters and a mass of 50 to $100 \mathrm{~kg}$. The crystallographic orientation is controlled by that of the silicon seed and it us usually following planes $\{100\}$ perpendicular to the direction of growth because this orientation makes it easy to texture the surface of the cells.

An alternative to the Czochralski method is the zone melting recrystallization technique $(\mathrm{Fz})$. In this case, a very high-purity silicon bar is melted locally by induction, while a single crystal of silicon is extracted from this molten zone which moves in the opposite direction. These materials are exceptionally pure, in particular with regard to the very low oxygen content, insofar as the liquid silicon is not in contact with any wall and therefore there is no contamination by the crucible. Fz-Si is generally used in R \& D where it has led to record efficiencies of crystalline silicon-based cells. The disadvantages of this technique for possible industrialization are, on the one hand, a higher production costs than those of $\mathrm{Cz}$-Si and, on the other hand, the need to start from high-purity silicon bars, in contrast to $\mathrm{Cz}-\mathrm{Si}$ which can be made from melted rocks.

2:2. Multicristalline silicon lingots. - In order to reduce manufacturing costs, major research efforts began in the 1970s on the production of multi-crystal ingots (see bottom of fig. 2). The pieces of polysilicon are poured and then melted in a crucible made of silicon nitride or silica, of parallelepiped shape coated with $\mathrm{SiO} / \mathrm{SiN}$. Controlled cooling of the bottom of the crucible causes directional crystallization (from bottom to top) and the formation of a silicon ingot composed of large grains whose size is a function of the speed and direction of crystallization. The micro-structure is generally columnar, with grains up to a length of 10 to $20 \mathrm{~cm}$ and a section of the order of $\mathrm{cm}^{2}$. This progressive 
solidification, which maintains in contact with a solid phase and a liquid phase, develops a segregation of the impurities contained in the charge, which are drained upwards from the molded ingot. Parallelepipedal ingots weighing several hundred kilograms (currently 300 to $600 \mathrm{~kg}$ ) are commonly prepared and are then cut into "bricks", which themselves are cut into plates.

The production of multi-crystalline silicon ingots is cheaper than the Czochralski ingots with less energy consumption. It does not require elaborate equipment, nor precise control of temperatures and speeds. Furthermore, since the process tolerates the basic impurities better, a less expensive silicon source can be used. The drawback is that the resulting material is of lower quality. The lifetimes of the carriers are lower and the intrinsic performances of the multicrystalline silicon-based cells are approximately $20 \%$ lower than those of monocrystalline silicon. This is due, among other things, to grain boundaries where impurities are accumulated, in addition to some crystal defects (dislocations) and the presence of metallic impurities.

2*3. Fabrication of silicon solar cells. - The basic material for the manufacture of the cells is a monocrystaline or multicrystamline silicon wafer, usually $p$-type, with a thickness of about $150-300 \mu \mathrm{m}$ and a dimension of $156 \times 156 \mathrm{~mm}^{2}$. The conventional manufacturing process can be broken down into several steps (fig. 3). It can be noticed that the manufacture of cells based on crystalline silicon uses to a large extent technologies originally developed for the electronics industry: screen printing machines, drying and annealing furnaces for the application of contacts. The main steps are the following:

i) Surface treatment and texturing: an acid attack on the surface of the platelets is made in order to eliminate the damage due to sawing and to texture the surface and thus improve the absorption of the incident light.

ii) Formation of the PN junction by thermal diffusion of the dopants: the emitter is obtained by a diffusion of phosphorus limited to $0.2-0.3 \mu \mathrm{m}$ of depth to create a $n^{+}$highly doped emitter.

iii) Opening of the junction by reactive ion etching $\left(\mathrm{CF}_{4}+\mathrm{O}_{2}\right)$.

iv) Deposition of an antireflective layer of hydrogenated silicon nitride $\left(\mathrm{SiN}_{x}: \mathrm{H}\right)$ on the front face. This deposition reduces the reflection coefficient in the center of the visible spectrum from $34 \%$ to about $8 \%$ and also serves as a hydrogen reservoir for passivating the emitter surface and the volume defects of the wafer.

v) Formation of the contacts by screen printing followed by a high-temperature annealing $\left(>800^{\circ} \mathrm{C}\right)$ : silver grid on the front and aluminum layer on the back.

24. Performances of silicon solar cells. - The efficiency of the cells obtained in industrial production according to the process described above is in the range of $18 \%$ for a monocrystalline substrate and $16.5 \%$ for a multicrystalline substrate. In the laboratory, the efficiencies obtained are much higher thanks to the use of microelectronics technologies. 


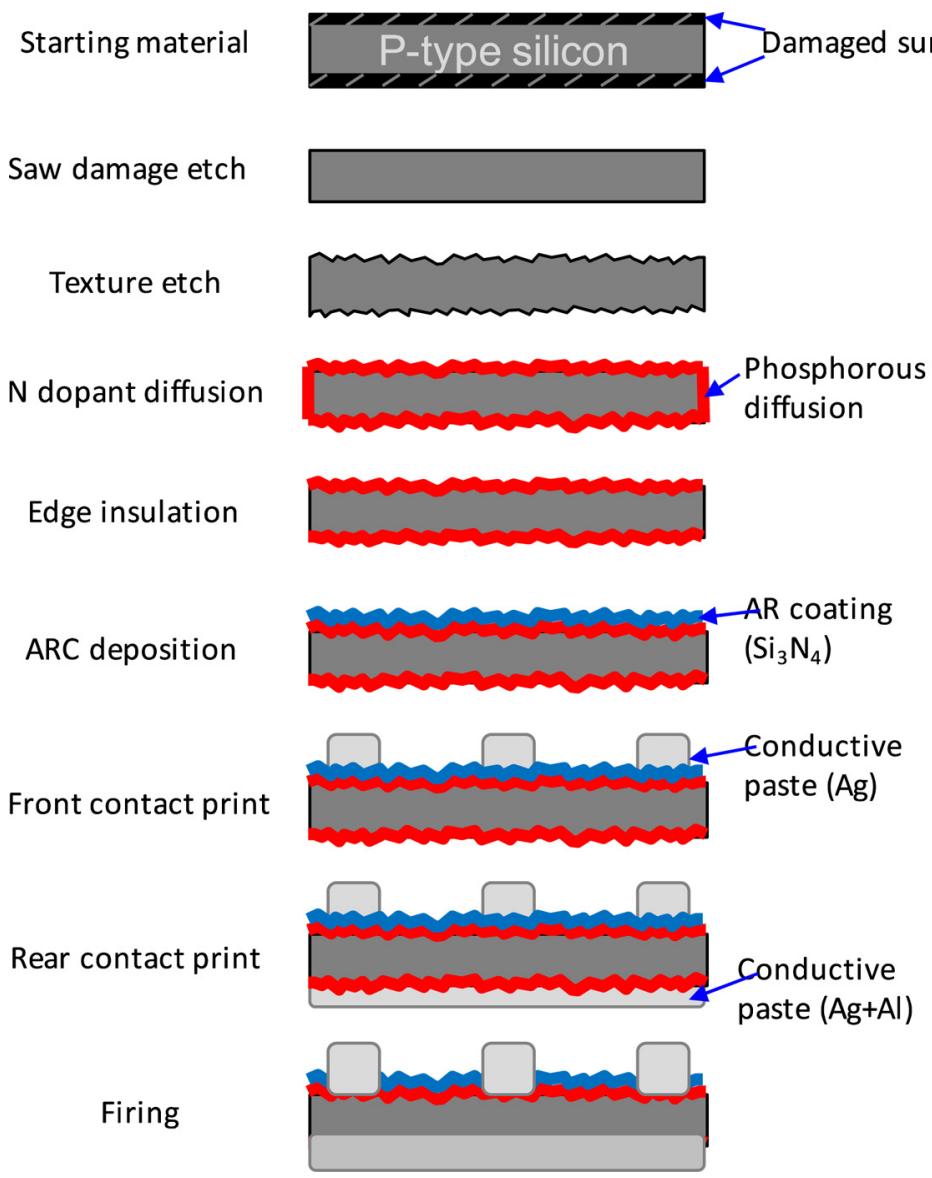

Fig. 3. - The different steps for the fabrication of a conventional silicon solar cell.

The world record for a silicon cell on a $p$-type substrate is held by the Center for Photovoltaic Materials and Systems at the University of New South Wales (UNSW) in Australia with a $24.7 \%$ Structure PERL (Passivated Emitter, Rear Locally diffused cell).

This structure made on a $p$-type FZ substrate (fig. 4) has several peculiarities compared to the standard process. The front is textured in reverse pyramids. The emitter is selective. A thin layer of thermal oxide is formed on the emitter to passivate the surface, and a double antireflection layer is deposited to reduce reflectivity. The back side is passivated by a thermal oxide which is then opened locally by chemical etching to make the contact. The contact region is previously doped $p^{+}$in order to reduce the contact resistances and to realize the BSF. It should also be noted that the doping on the front and rear surfaces is done by ion implantation, this technique allows a perfect control of the dopant profiles.

Considerable progress has been made in the manufacturing process over the last fifty years. The thickness of the wafers, which was $400 \mu \mathrm{m}$ in 1990, has gradually decreased to 


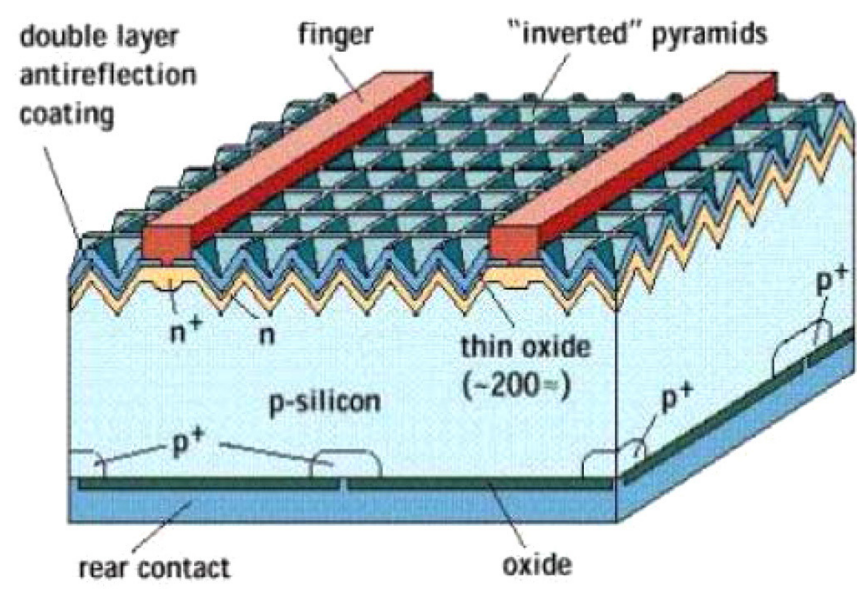

Fig. 4. - PERL structure as fabricated by University of New South Wales (Australia).

150-200 $\mu \mathrm{m}$ today. At the same time, the cell surface increased from $100 \mathrm{~cm}^{2}$ to $240 \mathrm{~cm}^{2}$. The output of screen-printed modules has increased from about $10 \%$ in 1990 to about $18 \%$ today for a monocrystalline substrate and $16.5 \%$ for a multi-crystal substrate, with the best modules exceeding 20\%. The annual production of manufacturing plants increases from 1-5 MWp in 1990 to several GWp. The learning curve of wafer-based silicon technology shows over the last thirty years that the price of modules has dropped by $20 \%$ on average for each doubling of installed capacity. This decline is due to the combined effect of technological innovation, market development and the emergence of Chinese industry. This cost can be further reduced because each step of the manufacturing process can be further improved by strengthening the automation of manufacturing units, in particular by pursuing the paths taken in previous years to reduce the costs of the industry:

- Reduction of the amount of silicon used.

- Use of $n$-type monocrystalline silicon at back junction or in heterojunction with amorphous silicon which achieves high efficiencies.

- Reduction or even suppression of the metallization of the front panel by means of rear junction cells and/or rear contacts.

2 4.1. Rear junction cells. Conventional solar cells have contacts on both sides. In the rear-junction cell based usually on $n$-type monocrystalline cells (also known as interdigitated contact cells), the two contact are placed on the rear face and form an interdigitated grid allowing the metallization of the front face and the concomitant shading to be completely eliminated. The junction is also on the rear side and the emitter and the $p^{+}$zones of the rear back surface field also form an interdigitated zone.

In conventional silicon cells, photogeneration occurs mainly on the surface. Here, the photogenerated carriers must therefore travel a distance which may be much greater than the thickness of the substrate to reach the junction at the rear of the cell. They can 


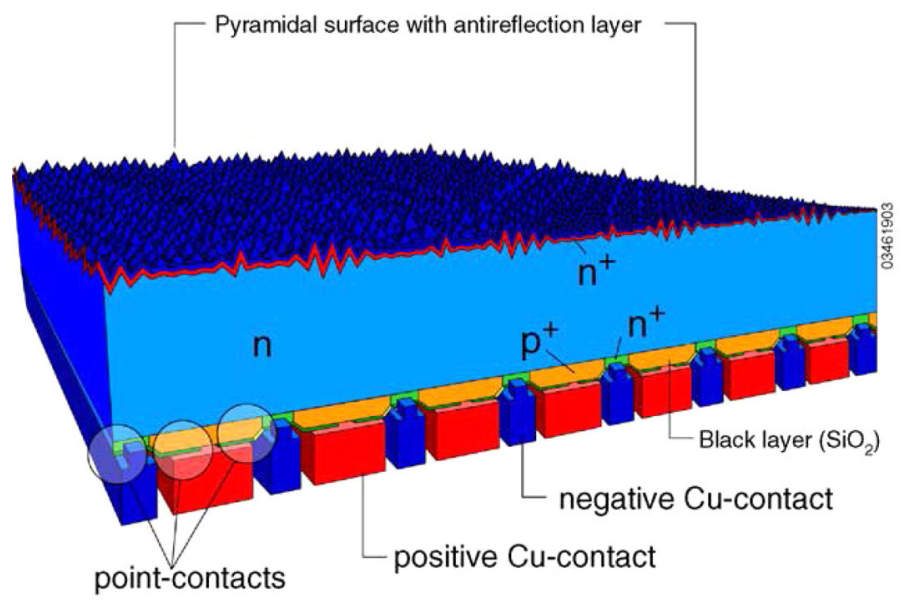

Fig. 5. - A "Sunpower" Interdigitated back contact cell based on type $n$-type silicon (source Granek).

recombine on the front if it is not well passivated or in the volume if their lifetime is not enough. The two most important parameters are thus the ratio of the diffusion length of the minority carriers to the thickness of the substrate, and the rate of recombination on the front face. Making a cell of this type therefore requires the use of high-purity monocrystalline silicon and high-quality manufacturing processes. They are reserved for uses where high efficiencies are suited. The rear junction cell A-300 marketed by Sunpower (fig. 5) was originally designed at Stanford University. It was marketed from 2004. In 2007, a new generation of A-300 cells reached an average yield of $22.4 \%$ in production. These cells have the highest production record. In 2010, a record $24.2 \%$ was achieved in $\mathrm{R} \& \mathrm{D}$.

2 4.2. Heterojunctions: bifacial HIT cell. The HIT (Heterojunction with Intrinsic Thin Layer) cell on an $n$-type monocrystalline silicon substrate was designed by Sanyo Electronic Corporation and marketed since 1997. Its principle is based on the deposition of a two-layer stack of hydrogenated amorphous silicon (An intrinsic layer and a doped layer) on each of the faces of the wafer by chemical vapor-phase (PECVD) at temperatures ranging from $200{ }^{\circ} \mathrm{C}$ to $400{ }^{\circ} \mathrm{C}$. The amorphous silicon forms a double tandem with the crystalline silicon (see fig. 6): On the front face, a $p$ - $i$-n heterojunction was formed by successive deposition of an intrinsic hydrogenated amorphous silicon (a-Si: H) layer on the $n$-type silicon and then a $p$-doped amorphous silicon layer. The intrinsic layer provides excellent passivation of the crystalline silicon while the doped layer acts as an emitter. This cell design eliminates the step of forming the emitter by diffusion of boron; on the rear, the same structure is found. The last layer of amorphous silicon is highly doped $n^{+}$, and acts as a back surface field. Given the low conductivity of the amorphous silicon, it is necessary to add a transparent conductive layer (TCO) in addition to the 


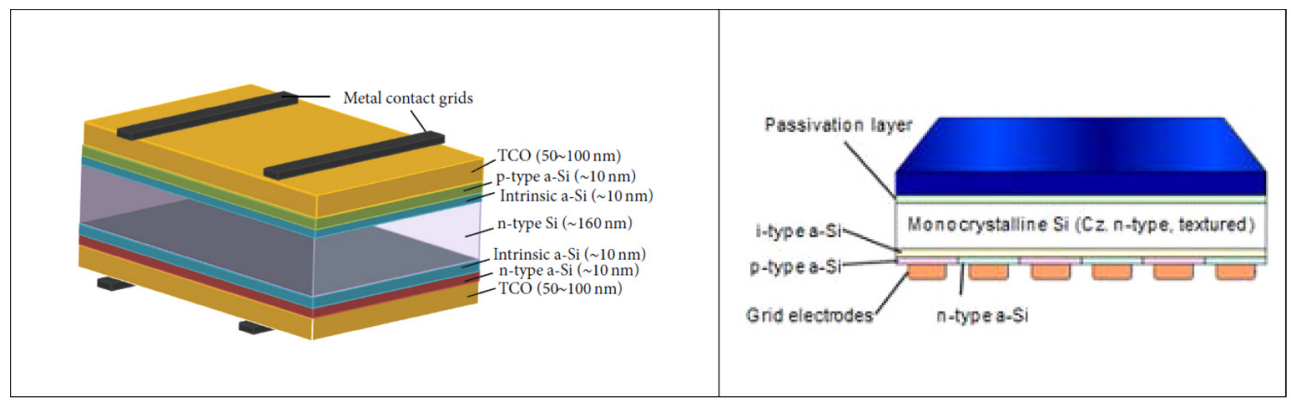

Fig. 6. - Schemes of a bifacial heterojunction cell (left) and back-contacts heterojunction cell (right).

silver collecting grids on each of the faces. This is illustrated in fig. 6 . The structure obtained is symmetrical and can be manufactured as a bifacial cell which can allow an improvement of the efficiencies by taking part of the light scattered at the rear of the modules.

The next challenges are to reduce the thickness of the crystalline silicon wafer to $100 \mu \mathrm{m}$ and to bring the contacts back to maximize the absorption of light. The efficiency world record on a heterojunction crystalline silicon cell $(26.3 \%)$ has been held since 2016 by the Japanese company Kaneka Corporation on a small area $\left(180 \mathrm{~cm}^{2}\right)$ cell with heterojunction on $n$-type substrate with back contacts.

25. Silicon photovolatic module. - The manufactured cells have a low open-circuit voltage $(0.6 \mathrm{~V})$ and are not useful as such. Modules are formed consisting of a number of solar cells of the same electrical characteristics which are interconnected (typically 72 in series) and encapsulated. A high voltage is thus obtained and the cells and their metallic contacts are protected from environmental stresses (moisture and hailstorms) and mechanical stresses (transport, assembly, wind) for a duration of at least 25 years.

The cells are generally connected in series in the module and are placed between 2 sheets of vinyl acetate (EVA), enclosed between two tempered glasses or between a glass plate and a tedlar sheet. A scheme of the module components is shown in fig. 7 .

The glass used on the front of the module is a tempered glass with a low iron content. It must have a high transmission coefficient in the wavelengths used by solar cells $(0.35 \mu \mathrm{m}$ to $1.10 \mu \mathrm{m}$ for silicon). The surface also needs little light reflection. In most cases, it ensures the mechanical strength of the module and the rigidity of the assembly. The glass also has the advantage of a perfect moisture tightness and a good self-cleaning power under the effect of rain. Its refractive index is close to 1.5, which is a good average between the air (index 1 ) and the cells (index 2 if they are provided with an antireflection layer). The two thin EVA sheets serve as a coating material and allow good adhesion between the cells and the front and rear faces of the module. The EVA has a good resistance to ultraviolet and temperature, a refractive index identical to that of glass and its implementation is relatively easy. The sandwich is laminated at $150{ }^{\circ} \mathrm{C}$, with the 


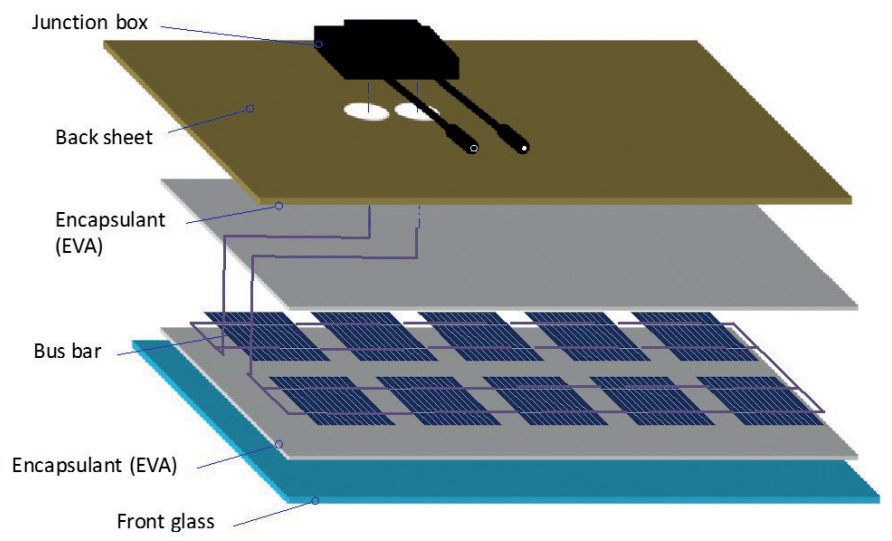

Fig. 7. - Scheme of a silicon module and its components.

aim of removing all the air from the module and crosslinking the EVA which will fill all interstices in order to give the material better resistance to temperature and UV.

The main function of the module's back is protection against moisture. Here a laminated sheet tedlar/aluminum/tedlar is generally preferred because lighter. Aluminum is a good barrier against moisture. One of the tedlar films isolates aluminum from the cells while the other protects aluminum from atmospheric conditions. The module is generally provided with an anodized aluminum frame which reinforces the strength and facilitates the fixing of the module on a structure. The back of the module is equipped with a connection box that contains the hot spot protection diodes and allows the module to be connected to the external circuit.

\section{3. - Polycrystalline thin-film modules (CIGS and CdTe)}

The family of thin-film compounds $(<5 \mu \mathrm{m})$ of crystalline semiconductors consists of direct band-gap materials that are highly visible and highly tolerant to defects $[11,12]$. The two most studied families are the CIGS chalcopyrites and the cadmium telluride (CdTe) [13-16]. The photovoltaic cells made with these materials are of the heterojunction type, that is to say that the $\mathrm{p}$ and $\mathrm{n}$ regions are made of different materials. The $p$-type semiconductor, $\mathrm{Cu}(\mathrm{In}, \mathrm{Ga})(\mathrm{Se}, \mathrm{S})_{2}$ (or CIGS) or CdTe, is the absorber of the cell (i.e. the area where the electron-hole pairs are photogenerated). The junction is formed with a cadmium sulphide (CdS) emitter, a broad band gap doped semiconductor. The absorption is minimized in the transmitter by the choice of a high band gap $\left(E_{g}(\mathrm{CdS})=2.42 \mathrm{eV}\right)$. This layer passes the visible radiation which is then absorbed into the absorber. The carriers are therefore only photogenerated in the absorber unlike the silicon where the n layer contributes to the photocurrent.

All these thin-film technologies have the advantage of being able to be deposited on rigid supports such as glass, or flexible materials such as stainless steel or plastic sheets (e.g., polyimides). The manufacturing processes used make it possible to avoid sawing 
of wafers and the inherent losses of raw material. Modules can be made into lightweight sheets or rolls and thus be more easily incorporated into building materials such as roofing elements, windows or facades. The conversion efficiencies obtained are still lower than those achieved with crystalline silicon, although significant progress has been made in recent years.

3·1. Chalcopyrities $(C I G S)$. - The technology called CIGS corresponds to the manufacture of thin-film modules using chalcopyrite-type ternary compounds as an absorbent layer: $\mathrm{CuInSe}_{2}$ (CIS), $\mathrm{CuInS}_{2}, \mathrm{CuGaSe}_{2}$ (CGS), with respectively bandgaps of $1.0 \mathrm{eV}$, $1.5 \mathrm{eV}$ and $1.7 \mathrm{eV}$, and their multi-alloys $\mathrm{Cu}(\mathrm{In}, \mathrm{Ga})(\mathrm{Se}, \mathrm{S})_{2}$ whose band gap can be made tunable depending on the composition. All these compounds have a direct band gap, which makes them suitable for use as an absorber in a thin-film photovoltaic cell because of their very high absorption coefficient (of the order of $10^{5} \mathrm{~cm}^{-1}$ ) in the visible range. This is compatible with the use of a thickness of 1 to 2 microns, about 100 times smaller than in the case of crystalline silicon. With this technology a record $22.6 \%$ efficiency has been achieved in the laboratory, while the typical yield of the marketed modules is in the order of 12 to $14 \%$.

The compounds $\mathrm{Cu}(\mathrm{In}, \mathrm{Ga})(\mathrm{Se}, \mathrm{S})_{2}$ belong to the so-called I-III-VI2 family. They crystallize in a structure derived from the Zinc-Blende structure. In the case of CIGS, this structure has an anionic sub-network (ions $\mathrm{C}_{2^{-}}$, e.g. $\mathrm{Se}_{2^{-}}$) and a cationic sub-network (ions $\mathrm{A}^{+}$and $\mathrm{B}^{3+}$, e.g. $\mathrm{Cu}^{+}$and $\mathrm{In}^{3+}$ ). The $\mathrm{CuInSe}_{2}$ structure, for example, can be deduced from the cubic zinc-blende structure of an II-VI material such as ZnSe in which the sites of the $\mathrm{Zn}^{2+}$ cations are alternately occupied by the $\mathrm{Cu}^{+}$and $\mathrm{In}^{3+}$ cations.

The CIGS can have an electrical conductivity of type $n$ or of type $p$, due to the presence of numerous intrinsic defects in the structure of the material. It is therefore possible to dope the CIGS with native defects without introducing extrinsic impurities as would be done with silicon. For example, an excess of selenium will lead to the $p$ type, a deficiency of selenium will lead to type $n$. The current photovoltaic cells use $\mathrm{Cu}(\mathrm{In}, \mathrm{Ga})(\mathrm{Se}, \mathrm{S})_{2}$ films prepared with an excess of Se and are therefore $p$-type with predominant hole concentrations between $10^{16}$ and $10^{17} \mathrm{~cm}^{-3}$ and diffusion lengths of the minority electrons of The order of the micron. In addition, CIGS-based cells are stable over time due to their resistance to radiation due mainly to the mobility of copper and the high density of defects, which are rearranged and ensure electrical stability.

The properties of CIGS (band gap and mesh parameters) can be varied by substituting some of the atoms of indium and/or selenium with atoms of the same valence ( $\mathrm{Ga}$ or $\mathrm{Al}$ for In, S for Se). We then have band gap energies that vary between $1.04 \mathrm{eV}$ for $\mathrm{CuInSe} \mathrm{S}_{2}$ and $2.4 \mathrm{eV}$ for $\mathrm{CuGaS}_{2}$.

Figure 8 (left) represents the bandgap as a function of the lattice constant parameter for different chalcopyrites. The best performances have been obtained by substituting a portion of the indium atoms with gallium atoms, resulting in materials of the formula $\mathrm{CuIn}_{1-x} \mathrm{Ga}_{x} \mathrm{Se}_{2}$ : an optimum yield is observed for $x=0.2-0.3$ with $E_{g}=1.12-1.20 \mathrm{eV}$ (fig. 8 (left)). Another way is to substitute selenium atoms with sulfur atoms, leading to materials whose bandgap varies between $1.04 \mathrm{eV}$ and $1.57 \mathrm{eV}$. 



Fig. 8. - Left: bandgap versus lattice constant for some I-III-VI ${ }_{2}$ chalcopyrites. Right: conversion efficiency of $\mathrm{CuIn}_{1-x} \mathrm{Ga}_{x} \mathrm{Se}_{2}$, versus bandgap; the optimal efficiency is for $x=0,3$.

The most used deposition techniques of the chalcopyrites active layer are coevaporation or reactive cathodic co-sputtering at high temperatures of the Cu-In-GaSe-S elements which allow good control of the crystal growth of the CIGS material. They currently make it possible to obtain the best cell efficiencies. Another one is the cold deposition, followed by annealing and selenization and/or sulfurization. This is the case for sputtering, electrodeposit, ink jet printing techniques, which require annealing in order to obtain an absorber with sufficient crystallinity and good optoelectronic properties. On the other hand, processes at atmospheric pressure are attractive because they potentially greatly reduce initial investment compared to vacuum processes. However, the obtained module efficiencies remain lower.

The classical structure of a thin layer cell of $\mathrm{Cu}(\mathrm{In}, \mathrm{Ga})(\mathrm{S}, \mathrm{Se})_{2}$ is described in fig. 9 (left). Figure 9 (right) shows a transverse section through a scanning electron microscope of a real device. The latter has five thin layers (fig. 9 (right)) deposited on a rigid soda-lime glass (1-3 mm thick), a metal or a flexible polymide substrate.

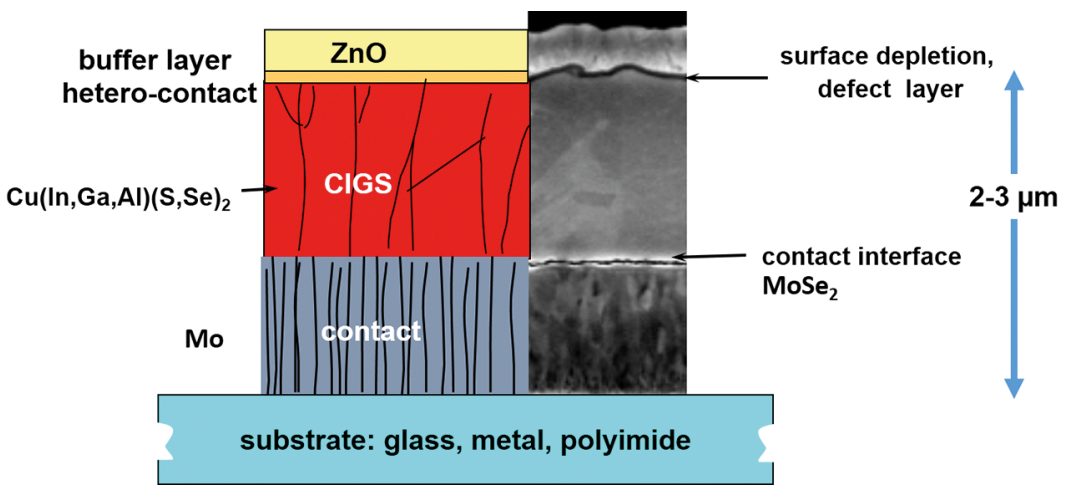

Fig. 9. - Left: scheme of a CIGS cell; right: MEB cross section of a CIGS structure (NREL). 


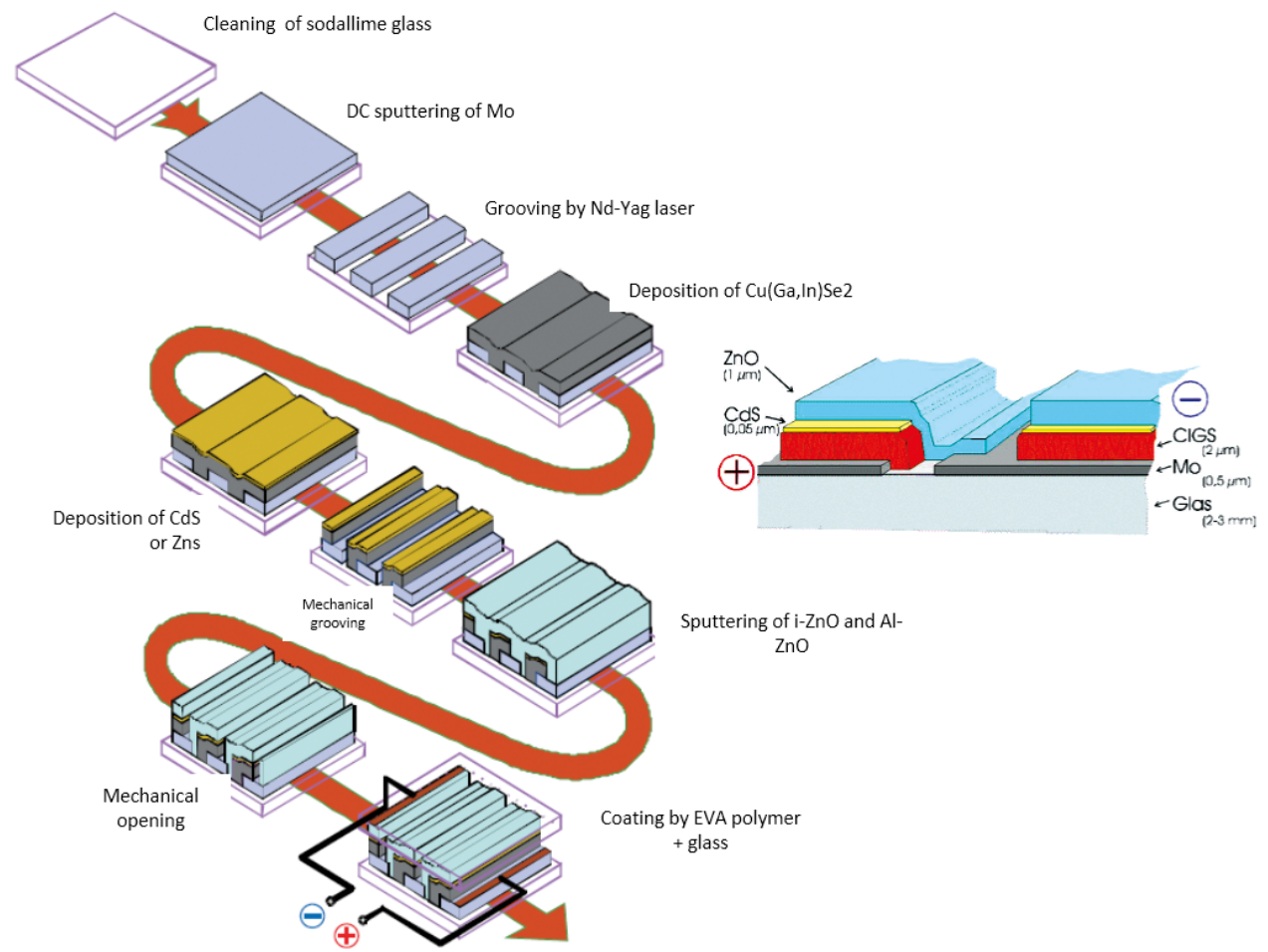

Fig. 10. - Left: steps for the fabrication of CIGS modules. Right: cross section of a CIGS cell.

The manufacture of CIGS modules differs from that of the conventional crystalline wafer silicon modules (fig. 10). The deposits are directly carried out on large surfaces (the sold modules have typically a surface area of $1.7 \mathrm{~m}^{2}$ ). In order to avoid excessive resistive losses and to achieve a high open-circuit voltage, a series of etchings is carried out after the layers have been deposited, in order to define cells and to put them in series by a monolithic interconnection method.

3.2. Cadmium telluride thin-film cells (CdTe). - Cadmium telluride (CdTe) is a II-VI compound that has a bandgap of $1.44 \mathrm{eV}$ and is therefore ideally suited for photovoltaic conversion. Its bandgap is of direct type and its absorption coefficient $\alpha(\lambda)$ for visible light is greater than $10^{5} \mathrm{~cm}^{-3}$. The absorbent layer therefore requires only 2 micrometers to absorb $99 \%$ of the solar spectrum without the need for sophisticated optical confinement.

Its absorption qualities and the wide variety of low-cost deposition techniques that can be used have made CdTe a particularly attractive material for the photovoltaic sector. CdTe's thin-film photovoltaic modules have been in development since the 1960s. They have been at the commercial stage since the mid-2000s. The American company First Solar is now the dominant player in this sector. 


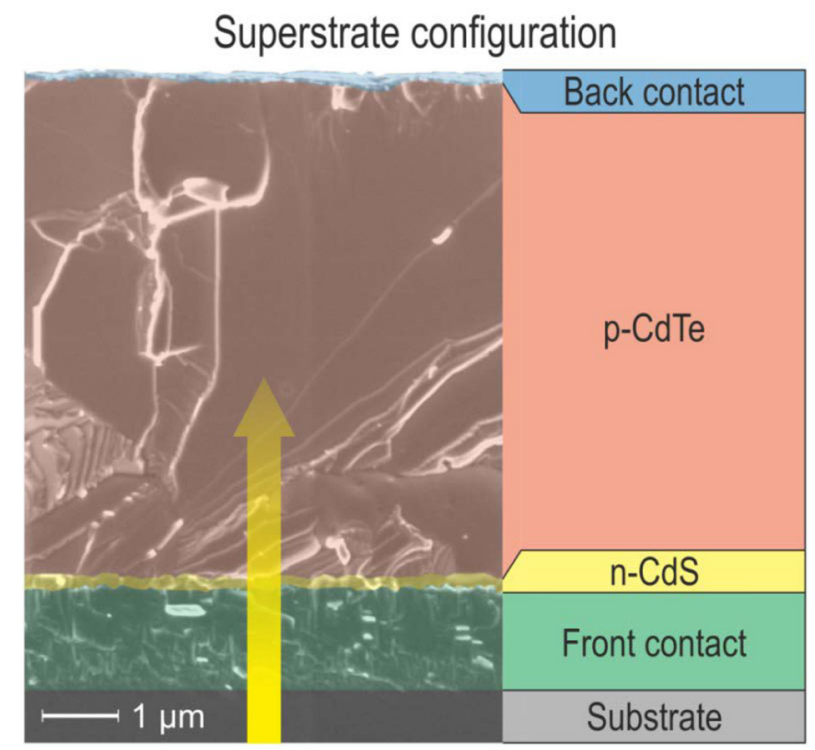

Fig. 11. - Left: MEB cross section of a CdTe based structure. Right: scheme of a CdTe cell in superstrate configuration.

A cross section of the CdTe cell structure is shown in fig. 11. The record laboratory yield was $22.1 \%$ while the average yield of the marketed modules was $16.1 \%$ at the end of 2015 .

One of the advantages of CdTe thin-film modules is that they can make a monolithic interconnection of cells so as to achieve a high open-circuit voltage. The steps are similar to those described above for the CIGS modules.

\section{4. - High-concentration photovoltaic systems}

The maturity of multi-junction cells (also called tandem) based on III-V compounds, which were developed for space applications from the 1990s, opened up new opportunities for terrestrial applications $[17,18]$. Given their very high cost $\left(\sim \$ 100.000 / \mathrm{m}^{2}\right)$, these cells are associated with a low-cost optical concentrator, such as Fresnel lenses, in order to significantly limit the amount of material used. This makes it possible to consider building large-scale photovoltaic power plants while limiting the total cost per unit area. However, high-resolution photovoltaic modules require a two-axis tracking system. Thus, they value mainly under direct illumination and can only be used in the arid and semiarid regions of the solar belt of the terrestrial globe, where direct illumination is high.

4*1. Multi-junction architecture and performance. - Multi-junction cells consist of a stack of several pn junctions in series. They are composed of semiconductor materials with increasing bandgap widths, each optimized for a different part of the solar spectrum. For a given number of junctions and a given illumination spectrum, there is an 
TABLE I. - Efficiency versus the number of junctions.

\begin{tabular}{|c|c|c|}
\hline Number of junctions & Efficiency under 1 sun & Maximum efficiency under concentration \\
\hline 1-junction & $33.7 \%$ & $40.8 \%$ \\
\hline 2-junction & $42.9 \%$ & $55.7 \%$ \\
\hline 3-junction & $49.3 \%$ & $63.8 \%$ \\
\hline$\infty$-junction & $68.2 \%$ & $86.8 \%$ \\
\hline
\end{tabular}

optimal choice of bandgap widths giving the highest efficiency: for example $33.7 \%$ for a homojunction, $42.9 \%$ for two junctions, $49.3 \%$ for three junctions, and $68.2 \%$ for an infinite stack of absorbers. This theoretical yield can reach $87 \%$ under high concentration (table I). In practice, the greater the number of junctions, the more complex the architecture and technology of the solar cell, and the less the efficiency increases.

The major components of III-V cells are gallium arsenide (GaAs) and indium phosphide (InP), which can be combined with other materials such as aluminum (Al) or tin (Sb) to forming ternary or quaternary alloys such as $\mathrm{Al}_{x} \mathrm{Ga}_{1-x} \mathrm{As}$ or $\operatorname{In}_{x} \mathrm{Ga}_{1-x} \mathrm{As}_{y} \mathrm{P}_{1-y}$. The growth of the layers is carried out by gas phase epitaxy from organometallic compounds. The elements used as dopants "N" are S, Se, Te, Sn while those used as dopants "P" are Zn, Be, Mg, Cd. The elements Si, C, Ge can act as dopants $n$ or dopants $p$ depending on whether they replace, respectively, a gallium (Ga) or arsenic (As) atom in the crystal structure $[19,20]$.

Figure 12 shows the relationship between the band gap and the crystal lattice parameter of several ternary and quaternary compounds as well as the transitions between them when the relative proportions of the elements in the compound are varied. This



Fig. 12. - Relationship between the bandgap and the lattice constant of several III-V compounds. 

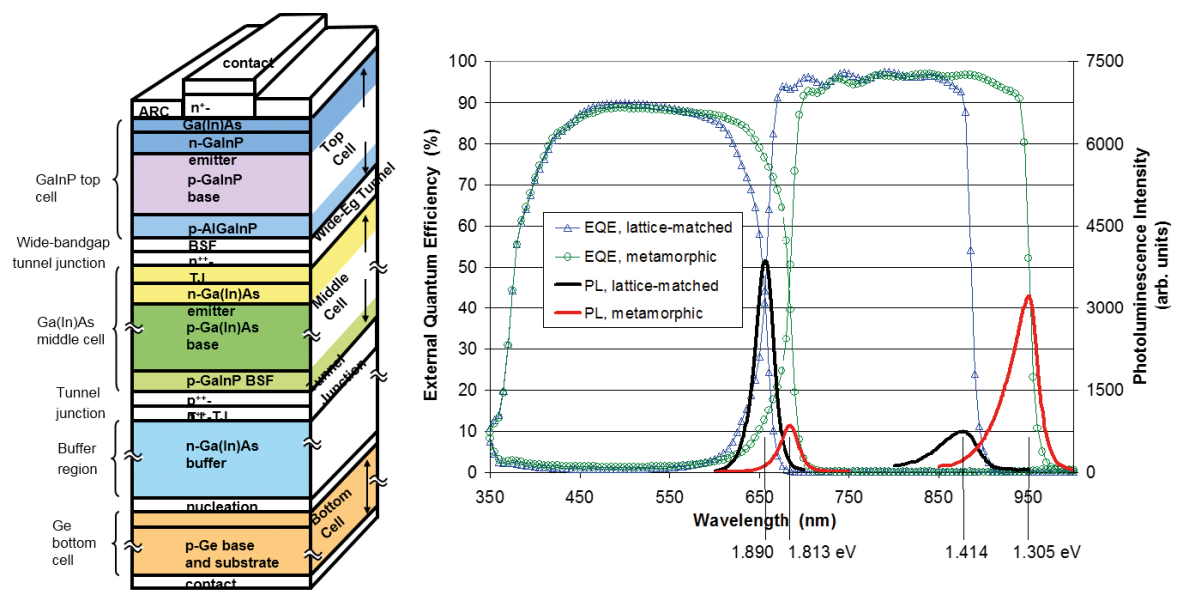

Fig. 13. - Left: scheme of a typical III-V tandem cell structure. Right: external quantum efficiency of a tandem cell.

diagram can be used to identify decreasing bandwidth semiconductors with lattice parameter' sizes (e.g., Ge, GaAs and AlAs have roughly the same lattice parameter with different band gaps). It also makes it possible to manufacture substrates having a gradient of lattice parameter in the case where the successive layers do not have suitable lattice constants (for example for a heterostructure between $\mathrm{GaP}$ and $\mathrm{GaAs}_{x} \mathrm{P}_{1-x}$ ). This is made possible by the low rate of growth of epitaxial techniques.

The triple junction cells marketed for terrestrial concentration systems are extremely sophisticated in their construction and use: they can have up to 60 epitaxial layers, 3 junctions and two tunnel diodes. Figure 13 (left) is exhibiting a typical tandem cell structure while fig. 13 (right) is showing the external quantum efficiency as expected from these structures.

As for fabricated cells, the highest efficiencies were obtained with structures based on stacks of III-V compounds, with direct bands and low temperature coefficients, manufactured by epitaxy. A record $46 \%$ concentration (508X) was achieved in 2016 in the laboratory (FhgISE, DE) for triple-junction III-V cells.

Due to the series connection, the current produced by the multi-junction cell is limited by the cell producing the lowest photocurrent. The consequence is that these cells are sensitive to changes in the solar spectrum that can occur during a day or a season and between different places.

Several geometries of lenses exist for the concentration purpose: curved cylindrical lenses with linear focus where the engravings are parallel and lenses with circular symmetry, planar or spherical. Planar lenses with circular symmetry are the most commonly used in high concentration (fig. 14(a)). The modules are composed of assembly of lenses and multijunction cells (fig. 14(b)). 




Fig. 14. - (a) Scheme of a planar lense that concentrates light on a multijunction cell. (b) Concentrated photovoltaic modules.

\section{5. - Prospects of medium and long-term technological breakdowns}

The technologies presented in the previous paragraphs are all commercial. The focus was on inorganic based photovoltaics using silicon wafers, CIGS or CdTe thin films or multijunction tandem cells. In the medium and long term, other channels are likely to create genuine technological breakthroughs that could lower the costs of the modules. Among the innovations expected, i) advanced architectures allowing to obtain very high efficiencies, thanks to relevant optical managements (down-conversion, up-conversion, plasmonics, ...), ii) thin organic or hybrid films (inorganic and organic combination) which can be manufactured at a very low cost, and iii) the emerging branch of perovskite cells [21-23], which is currently experiencing considerable enthusiasm in the scientific community and has demonstrated more than $20 \%$ efficiency in less than 5 years of investigations.

\section{REFERENCES}

[1] Shockley William and Queisser Hans J., "Detailed Balance Limit of Efficiency of P-n Junction Solar Cells", J. Appl. Phys., 32 (1961) 510; doi:10.1063/1.1736034.

[2] Hu Chenming and White Richard M., Solar Cells: From Basics to Advanced Systems (McGraw-Hill, New York) 1983.

[3] Goetzberger Adolf, Crystalline Silicon Solar Cells: Technology and Systems Applications (John Wiley \& Sons Ltd) 1998.

[4] Goetzberger Adolf and Volker U. Hoffmann, Photovoltaic Solar Energy Generation (Springer, Berlin [u.a.]) 2010.

[5] Green Martin A., "Lambertian Light Trapping in Textured Solar Cells and LightEmitting Diodes: Analytical Solutions", Prog. Photovoltaics: Res. Appl., 10 (2002) 235; doi:10.1002/pip.404.

[6] Green Martin A., Third Generation Photovoltaics Advanced Solar Energy Conversion (Springer, Berlin, New York) 2006. 
[7] Silicon and Ferrosilicon: Global Industry Markets and Outlook, 14th edition (Roskill) 2014, https://roskill.com/product/silicon-ferrosilicon/.

[8] Solanki C. S., Solar Photovoltaics: Fundamentals, Technologies and Applications (PHI Learning Private Limited) 2012.

[9] Markvart T. and Castañer Luis, Solar Cells Materials, Manufacture and Operation (Elsevier Science, Oxford) 2005.

[10] Pizzini Sergio, "Towards Solar Grade Silicon: Challenges and Benefits for Low Cost Photovoltaics", Sol. Energy Mater. Sol. Cells (PVSEC 18), 94 (2010) 1528; doi:10.1016/j.solmat.2010.01.016.

[11] Nakajima K. and Noritaka Usami (Editors), Crystal Growth of Si for Solar Cells, Advances in Materials Research, Vol. 14 (Springer Verlag, Berlin) 2009.

[12] Poortmans Jef and Arkhipov Vladimir (Editors), Thin Film Solar Cells: Fabrication, Characterization and Applications, Wiley Series in Materials for Electronic and Optoelectronic Applications (Wiley, Hoboken, NJ) 2006.

[13] Romeo A., Terheggen M., Abou-Ras D., Bätzner D. L., Haug F.-J., Kälin M., Rudmann D. and Tiwari A. N., "Development of Thin-Film Cu(In,Ga)Se2 and CdTe Solar Cells", Prog. Photovoltaics: Res. Appl., 12 (2004) 93; doi:10.1002/pip.527.

[14] Gorji Nima E., Perez Mauricio D., Reggiani Ugo, and Sandrolini Leonardo, "A New Approach to Valence and Conduction Band Grading in CIGS Thin Film Solar Cells", Int. J. Eng. Technol., 4 (2012) 573; doi:10.7763/IJET.2012.V4.435.

[15] Alonso M. I., Garriga M., Durante Rincón C. A., Hernández E. and León M., "Optical Functions of Chalcopyrite CuGaxIn1-xSe2 Alloys", Appl. Phys. A, 74 (2002) 659; doi:10.1007/s003390100931.

[16] Kodigala Subba Ramaiah, Cu(InGa)Se2 Based Thin Film Solar Cells (Academic Press, London) 2010, http://www.sciencedirect.com/science/book/9780123736970.

[17] KurTz S., Opportunities and Challenges for Development of a Mature Concentrating Photovoltaic Power Industry (revision) (National Renewable Energy Laboratory (NREL), Golden, CO) 2012, http://www.osti.gov/scitech/biblio/935595.

[18] Luque Antonio and Hegedus Steven, Handbook of Photovoltaic Science and Engineering, second edition (Wiley) 2011.

[19] Martí A. and Luque A., Next Generation Photovoltaics: High Efficiency through Full Spectrum Utilization (Taylor \& Francis) 2003.

[20] Andreev V. M., Grilikhes V. A. and Rumyantev V. D., Photovoltaic Conversion of Concentrated Sunlight (Wiley) 1997, http://eu.wiley.com/WileyCDA/WileyTitle/ productCd-0471967653.html.

[21] Green Martin A., Ho-Baillie Anita and Snaith Henry J., "The Emergence of Perovskite Solar Cells", Nat. Photon., 8 (2014) 506; doi:10.1038/nphoton.2014.134.

[22] Mingzhen Liu, Johnston Michael B. and Snaith Henry J., "Efficient planar heterojunction perovskite solar cells by vapour deposition", Nature, 501 (2013) 395 doi:10.1038/nature12509.

[23] Albrecht Steve, Saliba Michael, Correa Baena Juan Pablo, Lang Felix, Kegelmann Lukas, Mews Mathias and Steier Ludmilla et al., "Monolithic Perovskite/silicon-Heterojunction Tandem Solar Cells Processed at Low Temperature" Energy Environ. Sci. 9 (2016) 81; doi:10.1039/C5EE02965A. 